\title{
INFLUENCIA DE LA CALIDAD DE SERVICIO AL CLIENTE EN LOS HOTELES DE GUAYAQUIL
}

\author{
INFLUENCE ON THE QUALITY OF SERVICE \\ TO THE CLIENT AT GUAYAQUIL HOTELS
}

\section{EDISON CASTRO PACCHA', MÓNICA PACCHA SOTO²}

\begin{tabular}{cc}
\hline 1 & Universidad Agraria del Ecuador. ecastro@uagraria.edu.ec \\
2 & Universidad Agraria del Ecuador. mpaccha@uagraria.edu.ec
\end{tabular}

RESUMEN

La demanda de turistas nacionales e internacionales en Ecuador ha tenido gran impacto en los últimos años, lo que ha convertido al turismo en el segundo ingreso económico no petrolero del país. Esto ha motivado la necesidad de investigar la calidad en el servicio por parte de los empleados y personas encargadas de atención al cliente enfocado en los hoteles de tres estrellas (Categorización Internacional) y segunda categoría (Denominación en Ecuador por el Ministerio de Turismo) de la ciudad de Guayaquil. Para ello, se emplearon diferentes métodos durante la investigación, el deductivo que permitió los análisis de postulados, teoremas, leyes, principios de aplicación universal y de comprobada validez; y el de la observación en la realización de visitas para obtener una clara apreciación relacionada a las diferentes conductas y situaciones. Para la recolección de información se utilizaron las encuestas y entrevistas. El presente artículo fue elaborado a partir de los resultados obtenidos de la investigación titulada "Análisis de la influencia de la calidad del servicio, en la satisfacción de los clientes, en los hoteles de 3 estrellas en la ciudad de Guayaquil para diseñar un programa de mejoras en la calidad de servicio al cliente", en la que se pudo identificar un problema que afecta a los clientes en términos de carencia de calidad y servicio a los huéspedes que se alojan en los hoteles de Guayaquil. Ante esto, se presentan algunas recomendaciones que aportan al mejoramiento de la calidad del servicio hotelero.

PALABRAS CLAVE: Servicio al cliente, turismo, satisfacción, calidad, hotelería.
ABSTRACT
The impact in the last years, what it has converted to the tourism into the second not petroleum economic income of the country. This has motivated the need to investigate the quality in the service on the part of the personnel and persons in charge of attention to the client focused in the hotels of three stars (international Categorization) and the second category (Denomination in Ecuador for the Department of Tourism), of the city of Guayaquil.

For it, the different methods were used: deductive, because it allowed to come with the analyses of postulates, theorems, laws, beginning of universal application and of verified validity to apply it during the investigation. The method of the observation in the visits realization to obtain a clear appreciation related to the different conducts and situations. For the information compilation the poll and interview instruments were used.

The present article was elaborated from the obtained results of the titled investigation "Analysis of the influence of the service quality, in the clients satisfaction, in the hotels of 3 stars in Guayaquil to design a program of improvements of the quality of service to the client", in that it was possible to identify a problem that affects the clients in terms of quality lack and service to the guests who stay at the hotels of Guayaquil. Before this, there appear some recommendations that contribute to the improvement of the quality of the hotel service.

KEYWORDS: service to customer, tourism, satisfaction, quality, hotel 
INTRODUCCIÓN

Hoy en día el turismo, la hotelería y la restauración son algunos de los motores principales económicos que conducen a este país. Al igual que en todos los países del mundo, el turismo es una de las figuras principales y altas en la generación de empleo. Según la OIT (2016) "el turismo internacional constituye el $7 \%$ del total de exportaciones mundiales de bienes y servicios, porcentaje superior al $6 \%$ registrado en 2014 y el 30\% de las exportaciones de servicios" (p.1). Además, indica que las llegadas de turistas internacionales aumentaron de 25 millones en 1950 a 278 millones en 1980, 674 millones en 2000 y 1186 millones en 2015. Francia, los Estados Unidos de América, España, la República Popular China e Italia son los cinco principales destinos en cuanto a llegadas de turistas internacionales.

En 2015, el turismo supuso la creación directa de 2,5 millones de empleos nuevos en todo el mundo, hasta alcanzar un total de 108 millones de empleos directos (3,6\% del empleo mundial total y $3 \%$ del PIB total). (OIT, 2016, p.1).

En el caso ecuatoriano los datos de la demanda turística en los últimos años, sobre todo en el año 2016, muestran la importancia que tiene el gasto turístico realizado por los no residentes, es decir turistas extranjeros que visitan nuestro país; este representa el 1.5\% del PIB (MINTUR, 2016), lo que llevó a registrar 484.884 miles de empleos turísticos.

Así mismo, con relación a las llegadas de extranjeros a Ecuador se registraron en diciembre del 2017, 171.926 turistas (MINTUR, 2017). El posicionamiento del turismo en la economía ecuatoriana se sitúa en el año 2016, de 1.075, 5 USD millones de dólares, su balanza turística registra un saldo positivo de 292.0 USD millones de dólares (MINTUR, 2016).

Como se menciona anteriormente el turismo, la hotelería y la restauración contribuyen al crecimiento económico del país. La industria hotelera requiere de una cartera fija de clientes, que estos sean rentables, leales para que el negocio crezca y se mantenga dentro de un entorno competitivo.

En relación a la calidad se pretende mejorar en la atención, servicio, imagen de los hoteles de tres estrellas de la ciudad de Guayaquil, a los turistas nacionales y extranjeros; teniendo como meta la contribución a una mejor rentabi- lidad hotelera. Se considera otro aspecto importante como es el liderazgo, por ser la capacidad que debe poseer todo administrador para poder dirigir a un grupo humano determinado. El tema de recursos humanos también es considerado, porque es necesario que las personas encargadas de esta área tengan las suficientes competencias al momento de seleccionar el personal idóneo.

La satisfacción, percepción del cliente está en relación con las expectativas que tenga sobre los servicios que le ofertan, lo influyente sobre el precio, servicios, instalaciones, si cuenta con tecnología actual o cuenta con recursos muy antiguos, si el personal verdaderamente lo atiende bien y siente tener vocación por el servicio, si tienen o no compromiso con los clientes que llegan al hotel. Las empresas eficientes en general intentan complacer al cliente prometiéndoles solo lo que pueden ofrecer en cuanto a servicios de alojamiento, y cuando el cliente está hospedado, el hotel ofrece un valor adicional, y que termina superando las expectativas de los clientes a través de sus experiencias hoteleras.

Siempre es bueno empezar con la mejoría del cliente interno, es decir los empleados, mejorar sus condiciones de trabajo, sus sueldos y salarios, bonos, motivación adicional por el trabajo, que tengan vocación de servicio. Como segundo punto se puede lograr con un gran liderazgo o jefe de área que se encargue de entrenar al personal, que cuente con un marco de valores éticos como la honradez, respeto, honestidad, lealtad y amor a la empresa.

El empleo de tecnología moderna, es uno de los grandes retos del turismo. Hoy en día quien no la utilice no es competitivo en el mercado hotelero. De manera que es importante que, a través de los departamentos de marketing o relaciones públicas, se cuente con el servicio post venta, marketing en redes sociales y un plan de social media, para que el hotel se pueda promocionar, posicionarse como imagen en sitios especializados de alojamiento. Esto permitirá competir exitosamente con el resto de hoteles existentes.

La investigación que sirve de base al presente artículo se llevó a cabo para saber de qué manera influye la calidad del servicio, en los hoteles de 3 estrellas - segunda categoría en la ciudad de Guayaquil, para la satisfacción de los clientes en el 2014. Los resultados de la misma detectaron las falencias existentes: los servicios no cumplen los estándares de calidad percibidos por los clientes, en consecuencia el cliente está 
insatisfecho por su experiencia negativa; en este caso, genera poca afluencia de huéspedes en estos hoteles. Esto ha permitido que, en el presente artículo, se planteen recomendaciones de acuerdo a nuevas tendencias para mejorar la calidad y el servicio en estos establecimientos hoteleros.

Se espera que esta problemática existente en los hoteles de tres estrellas de la ciudad de Guayaquil, se pueda solucionar con la propuesta de algunos lineamientos porque cada día la hotelería, restauración y turismo están expuestos a grandes cambios y exigencias por parte de los turistas y clientes que se hospedan en estos establecimientos de servicio. Es importante que los administradores, gerentes generales o dueños del hotel, estén conscientes de que deben mejorar, contando con personal profesional, mínimo con estudios de tercer nivel universitario que tengan competencias altas, tanto personales como académicas; que el personal sea bilingüe, que hablen perfectamente el idioma inglés, considerado un idioma universal. Para ello, debe existir una capacitación constante para el buen desempeño laboral, contar con gente experta en recursos humanos y solución de conflictos.

Finalmente se espera que las recomendaciones planteadas en temas de atención y servicio al cliente, contribuyan al mejoramiento de los hoteles de tres estrellas de la ciudad de Guayaquil, y sirva de referencia para futuros estudios, investigaciones, aportes en cuestiones de satisfacción del cliente y calidad.

\section{MATERIALES Y MÉTODOS}

La falta de calidad en servicio y atención al cliente, además de la ausencia de rentabilidad en alojamiento hotelero sucede en la ciudad de Guayaquil en los hoteles de tres estrellas; existe un porcentaje ocupacional que fluctúa entre $80 \%$ - 90\% de las habitaciones de lunes a jueves y en cambio un $40 \%$ de las habitaciones los fines de semana. Esto puede ser resultado de la falta de interés en la captación de turistas extranjeros y nacionales, posiblemente fallas en el servicio, infraestructura hotelera, disponibilidad de servicios tecnológicos, así mismo falencias en los empleados, en la que todo el peso de la responsabilidad es por parte de los gerentes y dueños del hotel; generando que su capacidad máxima de alojamiento no se utilice en su totalidad.

El tipo de investigación que se llevó a cabo fue de carácter descriptivo porque permitió describir los factores que influyen en calidad de atención y servicio en los hoteles. El enfoque cualitativo permitió analizar las percepciones de la calidad del servicio de atención al cliente, gustos y preferencias de los turistas nacionales y extranjeros en los hoteles de tres estrellas - segunda categoría. En cambio, por medio del enfoque cuantitativo se midieron los niveles de satisfacción del huésped a través de las encuestas y entrevistas que se realizaron en la investigación de campo. El método deductivo fue utilizado con los análisis de postulados, teoremas, leyes, principios de aplicación universal y de comprobada validez para aplicarlo durante la investigación.

La muestra que se escogió fue de los hoteles de tres estrellas (Hotel de Alborada, Howard Johnson, Marcelius, Air Suites, Plaza Montecarlo, Airport, Garzota Inn) - segunda categoría de la ciudad de Guayaquil, del Catastro de Alojamiento proporcionado por la Coordinación Zonal cinco del Ministerio de Turismo, y donde la mayoría de los turistas nacionales y extranjeros vienen del terminal terrestre y aeropuerto de la ciudad de Guayaquil. Es decir, el enfoque estuvo dirigido a obtener información sobre los gustos de los clientes registrados en dichos hoteles mencionados. Para la investigación de campo se realizó el método de muestreo probabilístico tipo muestreo aleatorio simple, utilizando formularios aplicados a los huéspedes de los hoteles de tres estrellas - segunda categoría. Se realizaron así 100 encuestas a un promedio de 134 huéspedes alojados en la semana.

\section{DISCUSIÓN Y RESULTADOS}

El marketing es la administración de relaciones redituables con el cliente. La meta doble del marketing consiste en atraer a nuevos clientes prometiéndoles un valor superior y mantener y hacer crecer a los clientes actuales satisfaciendo sus necesidades. El capital basado en los clientes es la combinación total de los valores del tiempo de vida de todos los clientes actuales y potenciales de una empresa. Por lo tanto, se trata de una medida del valor futuro de la base de clientes de la compañía. Evidentemente, cuanto más leales sean los clientes redituables de la compañía, mayor será su valor para la empresa. El capital basado en los clientes podría ser una mejor medida del desempeño de una empresa que las ventas actuales o la participación en el mercado. Mientras que las ventas y la participación en el mercado reflejan algo pasado, el capital de clientes sugiere el futuro. (Kotler \& Armstrong, 2012, p. 21).

HelpScout, citado en Sainz (2015) estima que, 
El 86\% de los consumidores están dispuestos a abandonar completamente una marca cuando ésta les proporciona una mala experiencia. El 51\% de los consumidores solo da una oportunidad a la marca antes de renunciar a ella. Es cinco veces más caro conseguir un nuevo cliente que mantener al cautivo. La fidelidad con el producto constituye el $65 \%$ del total de las ventas seguras de las empresas. La mala calidad de los productos se manifiesta en los estados financieros de las empresas y en el recaudo fiscal de los países. (p.64).

La captación de nuevos clientes a través de un buen plan y trabajo de marketing, se verá reflejada dentro de una empresa, pero para llegar a nuevos clientes siempre hay que saber atraerlos, y es cuando se les ofrece algo adicional a lo que ellos esperan recibir en servicio. Hay que saber cuidar y mantener a los clientes actuales, porque de experimentar una mala atención en el servicio lo estaríamos perdiendo, y sería muy difícil volver a contar con su fidelidad; ya que ellos son el futuro de la empresa, es de ellos quien el negocio sobrevive y se mantiene activo en el mercado.

Una vez conocido el porcentaje existente ocupacional que fluctúa entre $80 \%$ - 90\% de las habitaciones de lunes a jueves y en cambio un $40 \%$ de las habitaciones los fines de semana, se seleccionaron los siguientes hoteles: Hotel de Alborada, Howard Johnson, Marcelius, Air Suites, Plaza Montecarlo, Airport, Garzota Inn. Se procedió a estructurar las diferentes preguntas en las encuestas, siendo los turistas nacionales quienes se alojan más frente a los turistas extranjeros, en estos hoteles de tres estrellas - segunda categoría de la Ciudad de Guayaquil.

ANÁLISIS

La primera pregunta se aplicó con el fin de conocer el motivo del hospedaje, el mayor porcentaje que arrojaron las encuestas es por negocios, y se lo puede constatar en la Tabla 1.

TABLA 1. MOTIVOS DE VIAJE

\begin{tabular}{lc|}
\hline RESPUESTA & PORCENTAJE \\
\hline Negocios & $50 \%$ \\
\hline Turismo & $20 \%$ \\
\hline Tránsito & $30 \%$ \\
\hline
\end{tabular}

La segunda pregunta realizada a los turistas se refiere a la calificación del hotel y sus instalaciones, cuyos porcentajes indican que hay que mejorar su imagen como empresa hotelera, según muestra la Tabla 2.

\begin{tabular}{|c|c|}
\hline RESPUESTA & PORCENTAJE \\
\hline Excelente & $40 \%$ \\
\hline Muy bueno & $34 \%$ \\
\hline Bueno & $17 \%$ \\
\hline Regular & $9 \%$ \\
\hline Malo & $0 \%$ \\
\hline
\end{tabular}

La tercera pregunta realizada a los turistas se refiere a la atención del personal de recepción, cuyos porcentajes indican que hay que mejorar según lo que se muestra en la Tabla 3.

\begin{tabular}{lc}
\hline TABLA 3. LA ATENCIÓN DEL PERSONAL DE RECEPCIÓN \\
\hline RESPUESTA & PORCENTAJE \\
\hline Excelente & $42 \%$ \\
\hline Muy bueno & $44 \%$ \\
Bueno & $12 \%$ \\
Regular & $1 \%$ \\
\hline Malo & $1 \%$ \\
\hline
\end{tabular}

La cuarta pregunta realizada a los turistas se refiere al servicio de restaurante. Solo un $32 \%$ indican que es excelente, por lo que se puede deducir que se necesitan mejorías en este tipo de servicio; lo cual es mostrado a continuación en la Tabla 4.

TABLA 4. EL SERVICIO DEL RESTAURANTE

\begin{tabular}{lc}
\hline RESPUESTA & PORCENTAJE \\
\hline Excelente & $32 \%$ \\
\hline Muy bueno & $35 \%$ \\
Bueno & $29 \%$ \\
Regular & $4 \%$ \\
\hline Malo & $0 \%$ \\
\hline
\end{tabular}

La quinta pregunta se refiere al servicio de la habitación. Analizando los porcentajes de opinión obtenidos se deduce que debe mejorarse en este tipo de servicio. Estos resultados son mostrados en la Tabla 5.

TABLA 5. EL SERVICIO DE LA HABITACIÓN

\begin{tabular}{|lc|}
\hline RESPUESTA & PORCENTAJE \\
\hline Excelente & $32 \%$ \\
\hline Muy bueno & $35 \%$ \\
\hline Bueno & $29 \%$ \\
\hline Regular & $4 \%$ \\
\hline Malo & $0 \%$ \\
\hline
\end{tabular}

En la sexta pregunta, se refiere al tipo de recomendaciones que harían los huéspedes a los hoteles, y las respuestas se concentraron en: dar informa- 
ciones turísticas, salones para conferencias con altas condiciones tecnológicas, implementación de rampas para personas con discapacidad, dominio y conocimiento del idioma inglés por parte del personal hotelero. Estos resultados se pueden constatar en la Tabla 6 .

\section{TABLA 6. RECOMENDACIONES DE HUÉSPEDES}

\begin{tabular}{lc}
\hline RESPUESTA & PORCENTAJE \\
\hline Dar información turística & $28 \%$ \\
\hline Salones para conferencias & $44 \%$ \\
$\begin{array}{l}\text { Implementar rampa para personas con } \\
\text { movilidad reducida }\end{array}$ & $26 \%$ \\
Que el personal conozca el idioma inglés & $9 \%$
\end{tabular}

En el análisis de los resultados, se pudo determinar que la mayoría de los huéspedes que llegan a estos hoteles son nacionales, y a partir de ahí en segundo lugar son los turistas extranjeros. El motivo principal por el que llegan a la ciudad de Guayaquil es por negocios. Los resultados de ambos tipos de huéspedes indican que hay errores en cuanto a atención y servicio de calidad por parte de los empleados que pertenecen a estos hoteles de tres estrellas.

Se pudo determinar que existen falencias en el cliente interno de la empresa por falta de capacitación, profesionalismo. La mayoría de los empleados son contratados sin contar con formación universitaria, ni tampoco con habilidades para el manejo del idioma inglés. Asimismo, en cuanto a infraestructura, se evidencia la falta de salones modernos para conferencias, eventos, congresos; restaurantes; central telefónica; e implementación de rampas para personas con discapacidad. Es notable la ausencia de liderazgo por parte de los jefes de área o administradores del hotel; determinando que ellos desempeñaban varios cargos al mismo tiempo.

Se nota en algunos casos un clima laboral no muy adecuado, por parte de los empleados y jefes; de lo cual el departamento de recursos humanos deberá asumir la responsabilidad. Quizás los empleados no cuenten con las habilidades necesarias para su desempeño y desconozcan las competencias laborales según las normas del Instituto Nacional Ecuatoriano de Normalización (INEN).

Existe también falta de promoción de estos hoteles, que es tarea del departamento de marketing. No todos cuentan con esta área. Con un buen plan de social media, a través de redes sociales, se lograría retener clientes actuales y captar nuevos clientes potenciales.
Podemos definir calidad como,

el conjunto de aspectos y características de un producto y servicio que guardan relación con su capacidad para satisfacer las necesidades expresadas o latentes (necesidades que no han sido atendidas por ninguna empresa pero que son demandadas por el público) de los clientes. (Vértice, 2010, p.2).

La calidad es el nivel de excelencia que la empresa ha escogido alcanzar para satisfacer a su clientela clave; representa, al mismo tiempo, la medida en que se logra dicha calidad. "La gestión total de la calidad es un planteamiento organizativo que consiste en intentar mejorar constantemente la calidad en todos los procesos, productos y servicios de la organización" (Vértice, 2010, p.2).

Hoy en día, la alta dirección de las empresas considera la tarea de mejorar la calidad de los productos y servicios como la prioridad número uno. En el futuro la mayor parte de los clientes no aceptarán o tolerarán productos de calidad media. A las empresas no les queda otra opción que aceptar la Gestión Total de la Calidad (CTC) si quieren competir de forma rentable. Como señalan algunos empresarios, la calidad es el mejor camino para poder escoger a los clientes, la mejor defensa contra la competencia extranjera y el único modo para apoyar el crecimiento y la rentabilidad. (Vértice, 2010, p.15).

La competitividad ayuda a generar rentabilidad y ventajas sobre otras empresas o países en el mercado o en otros ámbitos. La competitividad depende de la relación costocalidad de lo ofrecido, y por ello es preciso utilizar estrategias de producción, gestión o administración más eficientes e innovadoras. Si la competitividad está orientada al cumplimiento de los requisitos de los clientes y a exceder sus expectativas logrando su satisfacción, entonces la competitividad se apoya en la calidad de los productos o servicios y en el enfoque hacia el cliente. (Marcelino y Ramírez , 2014, p.4).

Como podemos notar, los autores anteriormente citados coinciden en la importancia de la calidad y atención en el servicio que se ofrece al cliente, ya que este factor es muy importante al momento de elegir un establecimiento ho- 
telero. El término calidad en el sector turístico involucra alta competitividad y genera mucha más rentabilidad frente a otras empresas hoteleras, por lo que hay que trabajar e innovar para mostrar un mejor servicio al huésped.

La evolución de la calidad durante su desarrollo se ha fortalecido gracias a las aportaciones y los estudios realizados por importantes autores tales como Ishikawa (1986), Deming (1989), Juran (1996), Crosby (1987) y Feigenbaum (1999), entre otros pioneros visionarios no menos notables. Ellos son quienes han conseguido con sus teorías, estudios, modelos y herramientas trascender en el tema de la calidad. A continuación algunas referencias:

La calidad en la actualidad es algo que las organizaciones persiguen a toda costa con métodos, avances tecnológicos, teorías, filosofías, o estrategias que les brindan una esperanza y oportunidad para alcanzar estándares de calidad de clase mundial, que es a lo que aspiran la mayoría de las empresas; sin importar el tamaño, es posible convertirse en una empresa de calidad al ser una pequeña sociedad bien organizada, con políticas y objetivos definidos, enfoque en el cliente, relaciones mutuamente beneficiosas con los empleados, la sociedad y la empatía con el medio ambiente. (Marcelino y Ramírez, 2014, p.4).

Deming considera que la calidad se consigue mediante el control estadístico de todos los procesos, no solamente los relacionados con el producto. Para lograrlo es necesaria la participación del personal para que pueda aportar mejoras. Deming resumen en 14 puntos la filosofía que ha de seguir la dirección de una empresa para lograr la calidad. Joseph Juran, observa que las actividades que llevan a una gestión total de la calidad necesitan además del control estadístico, un método de análisis de problemas y otro de gestión. Esto se resume en la siguiente trilogía: Planificación de la calidad, control de la calidad obtenida, mejora de la calidad. Philip Crosby para la implantación del proceso de calidad, recomienda una serie de acciones, sus 14 puntos. El ciclo PDCA (Plan-Do-Check-Act, es decir, Planificar-Desarrollar-ControlarActuar) fue ideado por Shewhart y divulgado por Deming, quien lo utiliza para explicar que la mejora continua en cada una de las actividades de la empresa ha de seguir un ciclo que se repita de forma ininterrumpida; de hecho también es conocido como el ciclo o rueda de Deming. (González, Domingo, y Pérez, 2013, p. 24).

El modelo Malcolm Baldrige establece que los líderes deben enfocar sus esfuerzos hacia una dirección estratégica y hacia los clientes, evaluar los resultados obtenidos e implementar estrategias en procesos primarios, alineación de objetivos, y máximo aprovechamiento de los recursos para mejorar el desempeño de la organización y lograr la satisfacción de los grupos de interés. Se basa en un sistema de liderazgo y planificación estratégica, enfocada en el cliente y el mercado, con apoyo de la medición de índices, parámetro y el benchmarking (evaluación comparativa con la competencia), como una manera de crear ventaja competitiva, su objetivo es establecer un conjunto de criterios utilizados para evaluar la calidad y excelencia organizacional, impulsados por la autoevaluación para identificar fortalezas y áreas de oportunidad dentro de una política. (Marcelino y Ramírez, 2014, p. 148).

Una buena gestión de la calidad en la empresa forma parte de las estrategias de negocios para mantener y elevar las ventajas competitivas. Cuando hablamos en estos términos hay que hacer mención a la calidad total que es la que se extiende a cualquier actividad que desarrolle la empresa. (Díaz, 2014, p. $60)$.

La calidad tiene estrecha relación con la producción de bienes o servicios. Así, una adecuada identificación de las necesidades de los clientes estimula una producción mayor que las satisfaga de manera que, entre mejor lo haga, mayor deba ser la producción y sus beneficios, del mismo modo en que un estímulo en la creación de necesidades ficticias genera un incremento en la producción. En consecuencia, el cliente es quien establece el nivel de calidad, y las empresas deben responder a estas necesidades, especialmente en el diseño y fabricación de sus productos y servicios. En un esquema de competencia libre la empresa que no responda a este mecanismo es rápidamente excluida del mercado, mientras que en la competencia regulada se demora más tiempo. (Álvarez, 2015, p.16). 
De acuerdo con los modelos de calidad que estos autores plantean, se podría implementar en los hoteles de tres estrellas, métodos, teorías y tecnologías aplicables a la calidad. Con la participación de todos los miembros de la empresa se puede mejorar el tema de calidad y trabajarlo por etapas: planificar, controlar, y mejorar el servicio y atención hacia el huésped.

Con el modelo de Malcolm Baldrige (1997), por ejemplo, los administradores de hoteles podrían emplear estrategias adecuadas para mejorar como empresa, hacer una evaluación frente a la competencia. Si los resultados son buenos se está por encima de la competencia, si son malos hay que mejorar a nivel gerencial. Todas estas acciones se realizan con el objetivo de satisfacer al cliente.

En este sentido, la calidad podría definirse como "la mejora continua de todas las actividades que realiza la empresa, a fin de alcanzar un nivel de excelencia que satisfaga las demandas y expectativas de sus clientes" (Arenal, 2016, p.36). Todo ello mediante la activa participación de los niveles gerenciales y de todos los trabajadores.

La calidad es el factor por el cual el cliente cada día es más exigente en cuanto al consumo o uso de un producto o servicio. Por lo tanto, los hoteles de tres estrellas deben ser muy precisos en mejorar la calidad del servicio hacia sus clientes, de lo contrario, estos preferirán la competencia.

Varias teorías, modelos de mencionados autores se usaron en el análisis del objeto de estudio. El modelo de Malcolm Baldrige (1997) se aborda en este artículo y se menciona el liderazgo como tema principal, que tienen que tener como competencia los actuales gerentes, administradores de hoteles, supervisores o jefes de área, de los hoteles de tres estrellas.

También que se enfoquen cuidadosamente con el cliente, mercado, para mejorar la calidad y atención en el servicio, logrando implementar los círculos de calidad, que consisten en conformar grupos pequeños de personas o equipos de trabajo orientados a solucionar problemas o proporcionar ideas para mejorar un área, departamento, o aspecto del hotel. Una vez aplicado, se mejorarán los procesos de servicios y se llegará a tener competitividad hotelera, permitiendo evaluar y obtener resultados a nivel de toda la empresa.

La satisfacción implica superar expectativas. Para los clientes, la satisfacción moviliza una emoción, y conlleva una comparación entre sus expectativas y el valor percibido del producto o servicio recibido. A su vez, el valor percibido está formado por la suma de las prestaciones del producto o servicio entregado, más las emociones derivadas de la relación con la organización; y todo ello dividido por la suma del precio pagado y las incomodidades e incertidumbre que supone relacionarse con la organización. (López, 2014, p.13).

En países desarrollados, hasta el $80 \%$ de los consumidores están dispuestos a pagar más a cambio de un mejor servicio al cliente. El $40 \%$ de los consumidores reclama a las marcas una mejor interacción con sus servicios de atención al cliente. El 73\% de los consumidores se queja por la mala atención de los empleados de mostrador y de su incompetencia. (Sainz, 2015, p.64).

Con los criterios de autores anteriormente citados y con el análisis de los resultados de las entrevistas y encuestas realizadas, se puede evidenciar la necesidad de mejorar la atención al cliente. Por eso, se enfatiza en la mejora de la calidad rediseñando los procesos y contando con personal profesional implementándolos acorde a lo que nos exige el cliente. Se pueden elaborar nuevos tratos con socios y alianzas estratégicas, promociones, que permitan mejorar la rentabilidad hotelera y lograr un programa de fidelidad con el cliente, ya que al contar con esto, el cliente es parte de la empresa, como si fuera familia de la misma; es decir tratar de complacerlo y satisfacer sus necesidades.

Los recursos humanos se deben disponer de evidencias de que el personal es lo suficientemente competente para realizar las tareas que se le asignan (estudios, capacitación, habilidades y experiencia). En la biblioteca, el trabajo de todas las personas incide, directa o indirectamente, en la calidad de los servicios prestados. (Balagué y Saarti, 2014, p.97).

Se puede afirmar que los empleados son los clientes más importantes para la dirección, especialmente, para aquellas empresas en las que la calidad del producto de los servicios y de la atención al cliente dependa del empleado. No se debe olvidar que los avances más espectaculares en productividad, en los servicios y atención al cliente se basarán 
más en la gestión que realicemos del personal, es decir, el marketing interno, que en su sustitución por máquinas. La selección de personal tendrá cada día más importancia para saber escoger aquellos empleados que tengan vocación y satisfacción por cada tipo de trabajo y, en general, por el trabajo bien hecho. Adecuar a cada estrategia de marketing externo y al perfil del puesto de trabajo la persona a ocupar, será condición básica para obtener el deseo de que el cliente quede satisfecho y repita. (Martínez, 2012 a, p.251).

En el caso de Recursos Humanos, "lo básico" será brindar procesos ordenados de Selección, Desempeño y Desarrollo, los tres pilares de un sistema de Recursos Humanos exitoso, tanto desde la mirada de la organización como del colaborador y del jefe. Los tres pilares mencionados, a su vez, se basan en las descripciones de puestos. (Alles, 2016, p.22).

Las acotaciones de algunos autores permiten notar la importancia del rol que desempeña el personal de recursos humanos, capital humano o talento humano. Por tanto, quienes ejercen esta función deben ser profesionales, con competencias de líder, capaces de dirigir y llevar a cabo con eficiencia todas las actividades inherentes a su cargo.

Son los empleados quienes están en contacto con el cliente externo. Por lo tanto, es competencia de recursos humanos o talento humano, que se dirijan bien y cuiden de él. Esto se puede llevar a cabo dándose un adecuado clima laboral, una clara motivación, y lo más importante una capacitación constante por parte de la empresa. Ello facilitará realizar una evaluación total a los empleados y permitirá comparar los resultados donde se vea si la capacitación fue efectiva para ellos y se vea reflejada durante su trabajo atendiendo al cliente.

El liderazgo es otro de los factores fundamentales en la administración de las empresas hoteleras abordado en este articulo. A través de éste se puede promover una mejor comunicación, creatividad, sentido de pertenencia, y el logro de los objetivos propuestos.

Liderar con el ejemplo:

Capacidad para comunicar la visión estratégica y los valores de la organización a través de un modelo de conducción personal acorde con la ética, y motivar a los colaboradores a alcanzar los objetivos planteados con sentido de pertenencia y real compromiso. Capacidad para promover la innovación y la creatividad, en un ambiente de trabajo confortable. (Alles, 2012, p.22).

Liderazgo ejecutivo o líder de líderes:

Capacidad para dirigir a un grupo o equipo de trabajo del que dependen otros equipos, y comunicar la visión de la organización, tanto desde su rol formal como desde la autoridad moral que define su carácter de líder. Implica ser un líder de líderes, al crear un clima de energía y compromiso junto con un fuerte deseo de guiar a los demás, que se verifica en el comportamiento de los otros al acompañar su gestión con entusiasmo. (Alles, 2012, p.37).

Liderazgo para el cambio:

Capacidad para comunicar la visión estratégica de la organización y lograr que la misma parezca no solo posible sino también deseable para los stakeholders. Capacidad para generar en los otros motivación y compromiso genuinos. Capacidad para promover la innovación y los nuevos emprendimientos, y lograr transformar las situaciones de cambio en oportunidades. (Alles, 2012, p.37).

El liderazgo se entiende como el proceso de influir en las actividades que realiza una persona o grupo para la consecución de una meta. El poder, a diferencia de liderazgo, es el potencial de influencia que desarrolla el líder; es el recurso que capacita al líder para inducir a los seguidores a que cumplan con la tarea u objeto. (Martínez, 2012, p.238).

Un gran líder sin lugar a dudas es con lo que debe contar toda empresa y en este caso un hotel de tres estrellas. El líder debe tener la capacidad y habilidad de dirigir, promover, motivar al personal con exigencia y respeto, de manera que éste se sienta comprometido con la empresa.

Otro aspecto importante que influye en la calidad del servicio es la infraestructura hotelera, las condiciones tecnológicas, el social media, etc. 
Todas las organizaciones necesitan una infraestructura para llevar a cabo sus actividades. Es necesario garantizar que los espacios de la biblioteca, mobiliario y equipo sean adecuados, y también que existan buenas condiciones del entorno de trabajo (p. ej.: iluminación, calefacción, ventilación, limpieza) y que están bien equipados con servicios de apoyo (p. ej.: teléfonos, redes). La biblioteca no puede prestar un buen servicio en locales con deficiencias de construcción, sin la iluminación o la calefacción adecuadas, o sin la infraestructura informática necesaria. (Balagué y Saarti, 2014, p.97).

La Tecnología se puede definir como el conjunto de conocimientos propios de un arte industrial, que permite la creación de artefactos o procesos para producirlos. Cada tecnología tiene un lenguaje propio, exclusivo y técnico, de forma que los elementos que la componen queden perfectamente definidos, de acuerdo con el léxico adoptado para la tecnología específica. (Cegarra, 2012, p.19). La aparición del Social Media promueve un cambio de paradigma organizativo en la comunicación y en las relaciones mantenidas por las personas a través de esos medios. Las aplicaciones de la 2.0 se basan en las redes sociales, en las herramientas colaborativas y en las comunidades basadas en entornos virtuales descentralizados, que permiten una mayor expansión e impacto del conocimiento, así como un acceso más sencillo, ágil e intuitivo de los usuarios. (Argos y Ezquerra, 2014, p.60).

Podemos hablar de dos acepciones, una de tipo técnica y otra de tipo formal: desde un punto de vista técnico, podemos decir que los Medios Sociales son todas las tecnologías, plataformas y herramientas desarrolladas a partir de la Web 2.0 que nos permiten interaccionar con otros usuarios, empresas o marcas. La otra, la formal, la más habitual cuando escuchamos el término, se refiere a todas las formas de compartir, comunicar, crear, gestionar, recibir, dar y obtener información y contenidos, y supone una democratización en cuanto a que da voz a cualquier individuo o entidad con capacidad para comunicar, y relevancia a aquellos que mayor destreza tengan para conseguirlo, a través de los diversos canales y redes. (Vela, 2014, p.23).
En una empresa hotelera si no hay las mejores condiciones de trabajo y servicio, lastimosamente no habrá clientes internos ni externos. Es por eso, que estos hoteles de tres estrellas deben tener la mejor infraestructura posible, instalaciones nuevas, modernas, con equipos, facilidades, accesorios nuevos, que atraiga a los clientes y tengan acceso al mismo.

Hoy en día la tecnología es el punto más fuerte y competitivo por la cual un cliente escoge donde alojarse. Es por eso, que hay que innovar en cada momento posible y es en este aspecto donde el hotel debe contar por ejemplo con WIFI, servicios de internet, salones para conferencia o diálogo, facilidades en general las 24 horas del día y sin ningún riesgo de que los clientes se queden sin este servicio.

Finalmente, se plantean algunas recomendaciones para minimizar estas fallas y contribuir a la rentabilidad hotelera en estos establecimientos ya mencionados.

\section{CONCLUSIONES Y RECOMENDACIONES}

Con los resultados obtenidos a través de las encuestas y entrevistas a los clientes extranjeros y nacionales se puede concluir que:

- Los clientes no están satisfechos con el servicio que brindan estos hoteles, requiriéndose de mejorarías, aplicando los círculos de calidad y atención de servicio.

- Existe ausencia de liderazgo por parte de los gerentes o administradores de los hoteles. Se necesita contar con líderes, empezando por los gerentes y luego por los responsables de cada departamento como: el de recursos humanos, de marketing, etc.

- Los empleados del hotel no cuentan con competencias necesarias, ni formación profesional, carecen de habilidades para el manejo del idioma inglés, falta formación en valores éticos que les permita un mejor desempeño; lo que impide ofrecer la adecuada información turística y atención que el cliente requiere. Se recomienda una capacitación constante, así como una selección minuciosa al momento de contratar el personal que va a laborar en la empresa.

- No existen salones modernos para conferencias, central telefónica, restaurantes, facilidad de acceso para discapa- 
citados, etc. Se necesitan implementar modernas y adecuadas instalaciones tanto en el aspecto de infraestructura como de tecnología. Todo esto contribuirá a una mejora en toda la organización hotelera y a obtener competitividad, rentabilidad y captación de nuevos clientes.

- No se dispone en la mayoría de los casos, de un departamento de Talento $\mathrm{Hu}^{-}$ mano, y si lo hay, el personal no se encuentra lo suficientemente capacitado para cumplir con todas las actividades que esta área requiere; una de ellas por ejemplo, la selección del personal que va a laborar en la empresa (de acuerdo a las normas INEN). El personal encargado de esta sección debe tener la suficiente formación profesional que lo habilite para un desempeño eficiente.

- Es necesario contar con un departamento de Marketing, en donde se haga énfasis en la elaboración de un plan social media para mejorar y promocionar los servicios que ofrece el hotel en internet. Ello puede hacerse a través de las redes sociales, que llegue a portales de preferencia y recomendación en alojamiento como Trip Advisor y Air Bnb Ecuador, además que cuente con servicios post venta para el cliente.

Se espera que estos lineamientos coadyuven al mejoramiento del servicio al cliente, a través de los círculos de calidad, nuevas tendencias y recomendaciones para minimizar falencias que de acuerdo a los resultados dados, impactan en la satisfacción de los huéspedes. Se han destacado temas como la falta de liderazgo, falta de infraestructura hotelera, competencias laborales INEN, falta de un departamento de marketing, mejora en los procesos de gestión de personal, capacitaciones, compromiso y sentido de pertenencia con la empresa hotelera.

\section{REFERENCIAS}

Alles, M. (2012). Social media y Recursos Humanos. Buenos Aires: Ediciones Cranica. Recuperado de http://site.ebrary.com/lib/uagrariaecsp/ reader.action?docID $=10862879$

Alles, M. (2016). Cuestiones sobre gestión de personas: qué hacer para resolverlas. Buenos Aires: Ediciones Granica. Recuperado de http:// site.ebrary.com/lib/uagrariaecsp/reader. action?docID=11217296
Álvarez, F. (2015). Calidad y auditoría en salud (3a.ed.). Bogotá: Ecoe Ediciones. Recuperado de http:// site.ebrary.com/lib/uagrariaecsp/reader. action?docID $=11162320 \& p p g=36$

Arenal, C. (2016). Calidad y servicios de proximidad en el pequeño comercio. Logroño: Editorial Tutor Formación. Recuperado de http:// site.ebrary.com/lib/uagrariaecsp/reader. action?docID=11200910

Argos, J., y EzQuerra, P. (2014). Liderazgo y Educación. Madrid: Editorial de la Universidad de Cantabria. Recuperado de http:// site.ebrary.com/lib/uagrariaecsp/reader. action?docID=10889818

BALAGuÉ, N., y SAARTI, J. (2014). Gestión dela calidad en la biblioteca. Barcelona: UOC. Recuperado de http://site.ebrary.com/lib/uagrariaecsp/ reader.action?docID $=10902454$

BALDRIGE, M. (1997). Baldrige national quality program. Washington: Addison-Wesley.

Cegarra, J. (2012). La tecnología. Madrid: Ediciones Díaz de Santos. Recuperado de http:// site.ebrary.com/lib/uagrariaecsp/reader. action?docID=11038764

Crosby, P. (1987). La calidad no cuesta. El arte de cerciorarse de la calidad. Colonia San Juan Tlihuaca: Compañia Editorial Continental, S.A. Recuperado de https://4grandesverdades.files. wordpress.com/2009/12/la-calidad-no-cuesta. pdf

DeMING, E. (1989). Calidad, productividad y competitividad. La salida de la crisis. Madrid: Díaz de Santos, S.A. Recuperado de https://books.google.com. ec/books?id=d9WL4BMVHi8C\&printsec=front cover\&dq=calidad+:+deming\&hl=es\&sa=X\&ve $\mathrm{d}=0$ ahUKEwjpoceFj-DZAhUKy1MKHUPKBDcQ

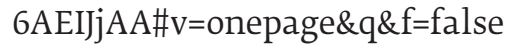

DíAz, M. (2014). Atención básica al cliente: manual teórico. Madrid: CEP, S.L. Recuperado de http:// site.ebrary.com/lib/uagrariaecsp/reader. action?docID=11126692\&ppg=10

Feigennaum, A. (1999). Control total de la calidad. Distrito Federal, México: Compañía Editorial Continental.

GonzÁlez, C., Domingo, R., y Pérez, M. (2013). Técnicas de mejora de la calidad. UNED - Universidad Nacional de Educación a Distancia. Recuperado de http://site.ebrary.com/lib/uagrariaecsp/ reader.action?docID=10803889

IsHIKAWA, K. (1986). ¿Que es el control total de calidad? La modalidad Japonesa. Bogotá: Grupo Editorial Norma. Recuperado de https://books.google. com.ec/books?id=MWGOXKteTQwC\&printsec $=$ frontcover\&dq=calidad+ishikawa\&hl=es\&sa= 
X\&ved=0ahUKEwizo8LykeDZAhWBzFMKHSH sDKUQ6AEIJjAA\#v=onepage\& $q \& \mathrm{f}=$ false

JurAN, J. (1996). Juranyla calidad por el diseño. Madrid: Díaz de Santos. Recuperado de https://books. google.com.ec/books?id=fURB60QH1RYC\&printsec=frontcover\&dq=calidad:+juran\&hl=es\& sa=X\&ved=0ahUKEwi-44ujk-DZAhVSzVMKHd veCKYQ6AEILDAB\#v=onepage\& $q$ f=false

Kotler , P., \& Armstrong, G. (2012). Marketing. México: Pearson Educación. Recuperado de https://profdariomarketing.files.wordpress. com/2014/03/marketing_kotler-armstrong. pdf

López, P. (2014). Cómo gestionar las reclamaciones de sus clientes. Madrid: AENOR - Asociación Española de Normalización y Certificación. Recuperado de http://site.ebrary.com/lib/uagrariaecsp/ reader.action?docID $=10862178$

Marcelino, M., y Ramírez, D. (2014). Administración de la calidad: nuevas perspectivas. México, D.F.: Patria. Recuperado de http://site.ebrary.com/ lib/uagrariaecsp/detail.action?docID=1101351 $1 \&$ p00=administracion+calidad

Martínez, M. (2012 a $)$. Calidad total y marketing interno. Madrid: Ediciones Díaz de Santos. Recuperado de http://site.ebrary.com/lib/uagrariaecsp/reader.action?docID=11038807

Martínez, M. (2012). Liderazgo. Madrid: Ediciones Díaz de Santos. Recuperado de http:// site.ebrary.com/lib/uagrariaecsp/reader. action?docID=11038597
MINTUR. (2016). Ministerio de Turismo. Indicadores turísticos. Recuperadodehttp://servicios.turismo. gob.ec/descargas/Turismo-cifras/Publicaciones/BoletinesMensualesTurismo/2017/02-INDICADORES_TURISTICOS-FEBRERO-2017.pdf

MINTUR. (2017). Ministerio de Turismo. Indicadores turísticos. Recuperado de http:// servicios.turismo.gob.ec/descargas/Turismocifras/Publicaciones/BoletinesMensualesTu rismo/2017/12-INDICADORES_TURISTICOSDICIEMBRE-2017.pdf

OIT (2016). Organización Internacional del Trabajo. Panorama del turismo. Recuperado el 24 de Julio de 2017, de http://www.ilo.org/wcmsp5/groups/ public/---ed_dialogue/---sector/documents/ publication/wcms_544242.pdf

SAINZ, R. (2015). UPGTO Management Review. doi:http://dx.doi.org/10.18583/umr.vli1.6.g12

Vela, D. (2014). Social media manager. Madrid: Difusora Larousse - Ediciones Pirámide. Recuperado de http://site.ebrary.com/lib/uagrariaecsp/ reader.action?docID $=11126496$

Vértice, P. (2010). Atención al cliente. España: Vértice. Recuperado de https://books.google.com. ec/books?id=SMcDP4U5z_AC\&printsec=frontc over\&dq=calidad+de+servicio+al+cliente\&hl= es-419\&sa=X\&ved=0ahUKEwiXsfCDm4_UAhU F6yYKHcThC9g4ChDoAQgtMAI\#v=onepage\&q \&f=false 